\title{
ANTIBIOTIC SUSCEPTIBILITY PROFILES OF MYCOPLASMA HOMINIS AND UREAPLASMA UREALYTICUM ISOLATED DURING A POPULATION-BASED STUDY CONCERNING WOMEN INFERTILITY IN NORTHEAST ROMANIA
}

\author{
Mareş Mihai $^{1 *}$, Năstasă Valentin ${ }^{1}$, Doroftei Bogdan², Chifiriuc Mariana Carmen ${ }^{3}$, Bleotu Coralia ${ }^{4}$, Socolov Demetra ${ }^{2}$ \\ ${ }^{1}$ Laboratory of Antimicrobial Chemotherapy, University “Ion Ionescu de la Brad”, Iaşi, Romania; "“Cuza Vodă” University \\ Hospital, 2nd Obstetrics-Gynecology Clinic, Iaşi, Romania; ${ }^{3}$ Faculty of Biology, University of Bucharest, Bucharest, Romania; \\ 4،Ştefan S. Nicolau” Institute of Virology, Romanian Academy, Bucharest, Romania.
}

Submitted: March 21, 2010; Approved: August 23, 2010.

\begin{abstract}
The study was carried out on 1068 infertile women under initial evaluation. For Mycoplasma hominis, the highest resistance rates were registered for ciprofloxacin $(72.22 \%)$, followed by macrolides and ofloxacin. For Ureaplasma urealyticum, the ciprofloxacin resistance was also high (51.72\%), while the resistance rates to other tested antibiotics were significantly lower.
\end{abstract}

Key words: Infertility, fluoroquinolones resistance, Mycoplasma hominis, Ureaplasma urealyticum

Infertility primarily refers to the biological inability of a person to procreate and, nowadays it is estimated that this condition affects approximately 72.4 million women worldwide (4). The childlessness has usually a lot of negative psychosocial consequences that may vary from fear, guilt, selfblame, marital stress, helplessness and depression to loss of social status, divorce or even violence-induced suicide (14).

The evaluation of an infertile individual comprise a broad panel of investigations - a detailed history, a complex physical examination and exhaustive laboratory analyses, in order to identify the main cause of the patient's impossibility to contribute to a child conception (11). Genital infections are common cause of infertility, often undiagnosed because of their non-specificity of clinical manifestations. Among the micro organisms involved in women's infertility, the literature cites the following bacterial species: Chlamydia trachomatis (CT), Ureaplasma urealyticum/Ureaplasma parvum (UU), Mycoplasma hominis (MH) and Neisseria gonorrhoeae (NG) (11). Both UU and $\mathrm{MH}$ are sexually transmitted bacterial pathogens undoubtedly implied in impairment of reproductive status, although numerous and often contradictory papers concerning their real pathogenic potential have been published last years. Spreading to other body areas is also possible, $\mathrm{MH}$ and UU being recovered from synovial fluid in high amounts up to $10^{7} \mathrm{CFU} / \mathrm{ml}(13)$.

Unlike conventional bacteria, $\mathrm{MH}$ does not have a rigid cell wall. Hence, they are not susceptible to penicillins and other antibiotics that act on this structure. They are, however, susceptible to a variety of other broad-spectrum antibiotics, most of which only inhibit their multiplication and do not kill

\footnotetext{
*Corresponding Author. Mailing address: Laboratory of Antimicrobial Chemotherapy, University „Ion Ionescu de la Brad” Postal address: 8 Aleea Sadoveanu, P4, 1st floor, 700489-Iaşi, Romania.; Tel.: +40 722465 789; Fax: +40 232407 316.; E-mail: mycomedica@gmail.com
} 
them. The tetracyclines have always been in the forefront of antibiotic usage, particularly for genital tract infections, but the newer macrolides, the ketolides and the newer quinolones have equal or sometimes greater activity. Mycoplasmas may be difficult to eradicate from human or animal hosts by antibiotic treatment because of resistance to the antibiotic, or because it lacks cidal activity, or because there is invasion of eukaryotic cells by some mycoplasmas. The quinolones also have the advantage of exhibiting some cidal activity (16). Fluoroquinolones are also attractive choices for treating genitourinary tract Ureaplasma infections. Data on antimicrobial resistance in ureaplasmas are very limited, because Ureaplasma spp. Cultures are rarely obtained for clinical purposes and in vitro susceptibilities are almost never performed. Different studies are showing that the level of resistance to doxycycline, josamycin, tetracycline, azithromycin, clarythromycin and pristinamycin is generally low, but the rate of resistance to fluoroquinolones (ofloxacin, ciprofloxacin) is showing an increasing rate in different studies. For example, Xie and Zhang reported $>50 \%$ resistance in a large number of strains isolated during 1999 and 2004 (19). Clinical isolates of fluoroquinolone-resistant Ureaplasma spp. have been also described thus far from France $(1,2)$ and USA (6). Duffy et al. reported the first case of naturally occurring fluoroquinolone resistance in Ureaplasma spp. from the United States, probably developed as a result of mutations in the gyrA and parC genes of the DNA gyrase/topoisomerase IV complex that occurred in the presence of antimicrobial selective pressure (6).

The purpose of this paper was to determine the antibiotic susceptibility profile of $\mathrm{MH}$ and UU isolated during a population-based study concerning women infertility in northeast Romania and to identify the most prevalent resistance markers in the respective strains.

\section{Patients}

The study has consisted in a screening of 1068 infertile women presented for initial evaluation in our outpatient clinic from May 2008 to September 2009. The median age of the patients enrolled in the study was 31 years (range 26-42). Approval for the study was granted by the Research Ethics Committee from the "Gr. T. Popa" University of Medicine and Pharmacy, Iasi (Romania). The study was conducted accordingly to the Declaration of Helsinki 2000.

\section{Sample collection}

Endocervical samples have been collected in duplicate from all patients using Chlamydia Swab/Brush Collection Kit (Bio-Rad Laboratories, France).

\section{Sample processing}

Soon after sampling, the swab has been processed using Mycoplasma IST2 kits (bioMérieux, France) in order to identify $\mathrm{MH}$ and UU, and to evaluate the susceptibility of the strains to 9 antibiotics, i.e. doxycycline, josamycin, ofloxacin, erythromycin, tetracycline, ciprofloxacin, azithromycin, clarythromycin, and pristinamycin.

\section{Bacteria detection}

The brush has been further submitted to DNA extraction using the DNA-Sorb-A kit (Sacace Biotechnologies, Italy). All extracted DNA samples were processed in an Applied Biosystems 7300 Real Time PCR system (Applera, USA) using the Mycoplasma hominis Real-TM and Ureaplasma urealyticum/Ureaplasma parvum Real-TM kits (Sacace Biotechnologies, Italy). The parameters of amplification were as follows: $95^{\circ} \mathrm{C}$ for $15 \mathrm{~min}$, followed by 10 cycles of $95^{\circ} \mathrm{C}$ for $20 \mathrm{~s}, 65^{\circ} \mathrm{C}$ for $20 \mathrm{~s}$ and $72^{\circ} \mathrm{C}$ for $20 \mathrm{~s}$, with a last stage of 35 cycles of $95^{\circ} \mathrm{C}$ for $25 \mathrm{~s}, 60^{\circ} \mathrm{C}$ for $30 \mathrm{~s}$ and $72^{\circ} \mathrm{C}$ for $15 \mathrm{~s}$.

After incubation of Mycoplasma IST2 strips, the positive samples have been registered and compared with the results obtained after RT-PCR assay in order to choose only the positive samples for $\mathrm{MH}$ and/or UU in the two tests. After the samples processing, we have selected 80 positive samples for 
MH and 372 for UU, respectively. For these samples, the susceptibility profiles to the mentioned above antibiotics were analyzed using the manufacturer recommendations.

There were considerable differences in levels of resistance to the antibacterial agents for the two bacterial species. However, the MH strains showed generally higher resistance rates than $\mathrm{UU}$ ones. For $\mathrm{MH}$, the highest resistance rates were registered for ciprofloxacin $(77.27 \%)$, followed by macrolides (azithromycin $38.88 \%$, clarythromycin and erythromycin $33.33 \%$ each) and ofloxacin (27.77\%). Lower resistance rates $(p=0.028)$ were registered for tetracycline, josamycin, pristinamycin, and doxycycline, i.e. $16.66 \%, 11.11 \%, 11.11 \%$, and $11.11 \%$ respectively (Fig. 1). For UU isolates, the ciprofloxacin resistance was also very high $(51.72 \%)$, while the resistance rates to the other tested antibiotics were significantly lower $(p=0.022)$, i.e. ofloxacin $(16.09 \%)$, erythromycin $(16.09 \%)$, clarythromycin $(9.19 \% \%)$, azithromycin $(8.05 \%)$, tetracycline $(5.75 \%)$, pristinamycin $(3.45 \%)$, josamycin $(2.30 \%)$ and doxycycline $(2.30 \%)$ (Fig. 1).

During this study we have reviewed the resistance rates of $\mathrm{MH}$ and UU isolated from genital swabs taken from women with infertility problems over the period from May 2008 to September 2009. These bacteria are members of the class Mollicutes, commonly referred to as mycoplasmas. The Mollicutes are the smallest known free-living microorganisms. $\mathrm{MH}$ is involved in the etiology of salpingitis and pelvic inflammatory disease, but its occurrence in sexually active population is lower than $\mathrm{UU}$, with an average of $10 \%(3,11$, 17). Thus, UU is the most common bacteria of the human urogenital tract (with a detection rate of $67 \%$ in sexually active women and $50 \%$ in men, respectively) that can cause lower pregnancy rates after in vitro fertilization, higher abortion rate of spontaneous pregnancies, increasing of the risk of premature contractions and preterm delivery, puerperal endometritis, orchitis, epididymitis, spermatocystitis, prostatitis, urethritis, increased apoptosis in human spermatogens, impairment of semen parameters, less stable chromatin and DNA denaturation in spermatozoa $(5,7,8,10,12,15,18)$.

Although mycoplasmas evolved from Gram-positive ancestors, the mycoplasmas lack a cell wall and are usually treated with quinolones, tetracyclines, or erythromycin. However, the number of resistant $\mathrm{MH}$ and $\mathrm{UU}$ strains is increasing every year following the widespread use of these agents.

The susceptibility rates showed that there was a considerable difference in levels of resistance to various antibacterial agents, and that the rate of change was related to the degree of antibacterial use. Doxycycline is still highly effective against MH and UU. Erythromycin, which is one of the most widely used antibiotics elsewhere, demonstrated high resistance rates in $\mathrm{MH}$. Ciprofloxacin, which was commonly used in treating patients with diarrhoea and other infections has demonstrated a high resistance rate in both tested microorganisms. The high resistance rates to ciprofloxacin observed in our strains could be indeed correlated with the antibiotic treatment history in the analyzed patients. Because the history of the patients reported a treatment with fluoroquinolones in the last 12 months (ciprofloxacin in 68 cases, ofloxacin in 56 cases, both in 28 cases and norfloxacin in 4 cases), it is likely that $\mathrm{MH}$ and UU isolates could become resistant to these antibiotics used for the treatment of other bacterial infections. For Romania, the explanation of this high resistance percentage to fluoroquinolones occurred in $\mathrm{MH}$ and UU strains isolated from human patients is the frequent prescription of these drugs by the general practitioners for the treatment of urinary and respiratory tract infections, pneumonia or otitis, due to their reduced price and lack of side reactions. The mechanism of resistance is probably the occurrence post exposure to fluoroquinolones, of a target alteration located in the DNA gyrase and topoisomerase IV subunits $(1,9)$.

Concluding, fluoroquinolones resistance was very high among MH and UU isolates, while macrolides resistance was low in UU and high in MH. Doxycycline was active against both organisms, exhibiting a low percentage of resistance. 
These results highlighted the emergence of antibiotic resistance in genital mycoplasmas from northeast Romania. Thus, the treatment of such infections should be guided by antibiotic susceptibility testing and local antibiotic resistance pattern.

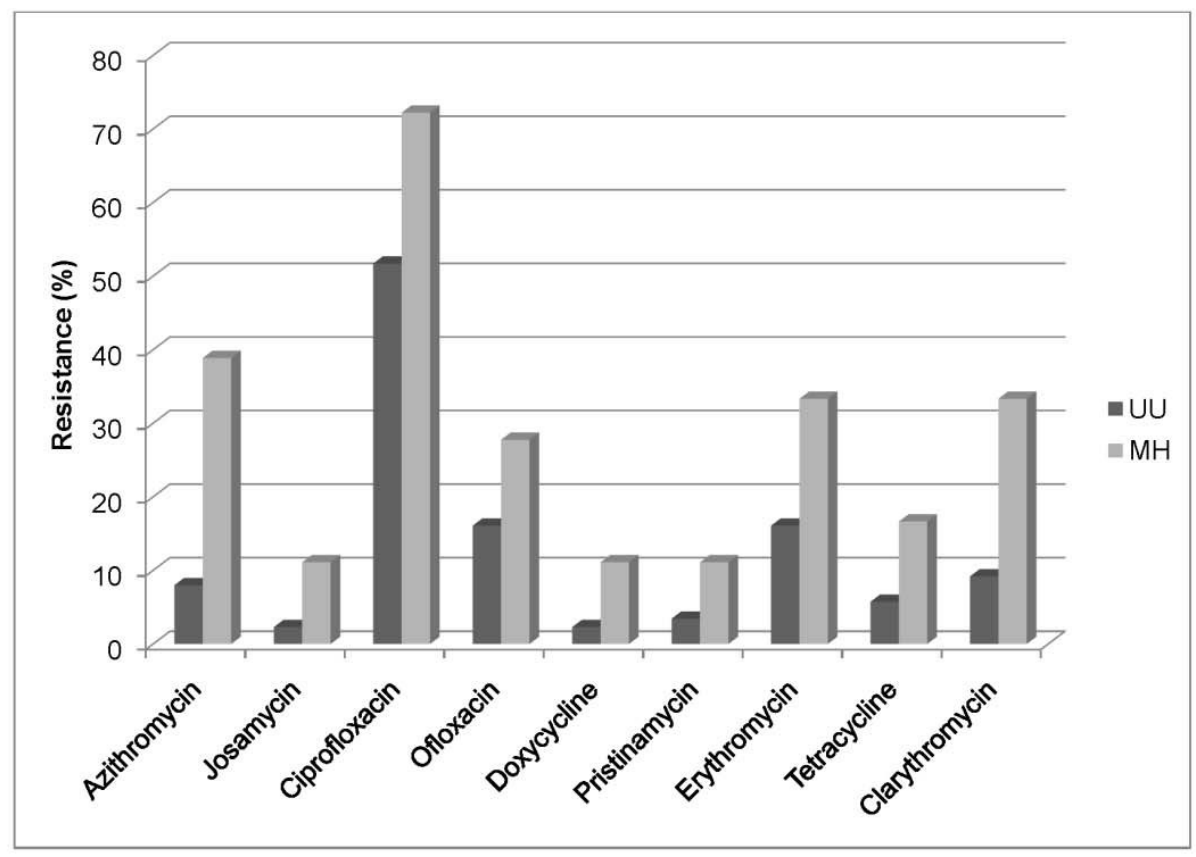

Figure 1. Resistance of Mycoplasma hominis and Ureaplasma urealyticum strains to the antibacterial agents

\section{ACKNOWLEDGMENTS}

We thank Gabriela Anton, Anca Botezatu and Cristina Daniela Goia from "Şt. Nicolau” Institute of Virology for their excellent technical assistance in performing the RT-PCR assays.

\section{REFERENCES}

1. Bébéar, C.M.; Renaudin, H.; Charron, A.; Clerc, M.; Pereyre, S.; Bébéar, C. (2003). DNA gyrase and topoisomerase IV mutations in clinical isolates of Ureaplasma spp. and Mycoplasma hominis resistant to fluoroquinolones. Antimicrob. Agents Chemother. 47, 3323-3325.

2. Bébéar, C.M.; Renaudin, H.; Charron, A.; Gruson, D.; Lefrancois, M.; Bébéar, C. (2000). In vitro activity of trovafloxacin compared to those of five antimicrobials against mycoplasmas including Mycoplasma hominis and Ureaplasma urealyticum fluoroquinolone-resistant isolates that have been genetically characterized. Antimicrob. Agents Chemother. 44, 25572560 .

3. Bébéar, C.M. (2002). Infections à mycoplasmes génitaux. In: Bébéar, C. (ed). Mycoplasmes et chlamydiae. Elsevier, Paris, France, p.37-47.

4. Boivin, J.; Bunting, L.; Collins, J.A.; Nygren, K.G. (2007). International estimates of infertility prevalence and treatment-seeking: potential need and demand for infertility medical care. Hum. Reprod. 6, 1506-1512.

5. Donders, G.G.; Bulck, van B.; Candron, J.; Londers, L.; Vereecken, A; Spitz, B. (2000). Relationship of bacterial vaginosis and Mycoplasma to the risk of spontaneous abortion. Am. J. Obstet. Gynecol. 183, 431-437.

6. Duffy, L.; Glass, J.; Hall, G.; Avery, R.; Rackley, R.; Peterson, S.; Waites, K. (2006). Fluoroquinolone resistance in Ureaplasma parvum in the United States. J. Clin. Microbiol. 44, 1590-1591.

7. Fowlkes, D.M.; MacLeod, J.; O'Leary, W.M.T. (1975). Mycoplasmas and human infertility: correlation of infection with alterations in seminal parameters. Fertil. Steril. 26, 1212-1218.

8. Gerber, S.; Vial, Y.; Hohlfeld P.; Witkin, S.S. (2003). Detection of Ureaplasma urealyticum in second trimester amniotic fluid by polymerase chain reaction correlates with subsequent preterm labor and 
delivery. J. Infect. Dis. 187, 518-521.

9. Gruson, D.; Pereyre, S.; Renaudin, H.; Charron, A.; Bébéar, C.; Bébéar, C.M. (2005). In vitro development of resistance to six and four fluoroquinolones in Mycoplasma pneumoniae and Mycoplasma hominis, respectively. Antimicrob. Agents Chemother. 49, 1190-1193.

10. Guillet-Rosso, F.; Fari, A.; Taylor, S.; Forman, R.; Belaisch-Allart, J.; Testart, J. (1987). Systematic semen culture and its influence on IVF management. Br. J. Obstet. Gynaecol. 94, 543-547.

11. Imudia, A.N.; Detti, L.; Puscheck, E.E.; Yelian, F.D.; Diamond, D.P. (2008). The prevalence of ureaplasma urealyticum, mycoplasma hominis, chlamydia trachomatis and neisseria gonorrhoeae infections, and the rubella status of patients undergoing an initial infertility evaluation. J. Assist. Reprod. Genet. 25, 43-46.

12. Montagut, J. M.; Lepretre, S.; Degoy, J.; Rousseau, M. (1991). Ureaplasma in semen and IVF. Hum. Reprod. 6, 727-729.

13. Oliveira Nascimiento de, C.M.; Figueiredo, C.A.; Timenetsky, J. (2002). Sensitivity of rabbit fibrochondrocytes to mycoplasmas. Braz. J. Microbiol. 33, 243-246.

14. Ombelet, W.; Cooke, I.; Dyer, S.; Serour, G.; Devroey, P. (2008). Infertility and the provision of infertility medical services in developing countries. Hum. Reprod. Update. 6, 605-621.
15. Reichart, M.; Kahane, I.; Bartoov, B. (2000). In vivo and in vitro impairment of human and ram sperm nuclear chromatin integrity by sexually transmitted Ureaplasma urealyticum infection. Biol. Reprod. 63, 1041-1048.

16. Taylor-Robinson, D.; Bébéar, C. (1997). Antibiotic susceptibilities of mycoplasmas and treatment of mycoplasmal infections. J. Antimicrob. Chemother. 40, 622-630.

17. Waites, K.B.; Schelonka, R.L.; Xiao, L.; Grigsby, P.L.; Novy, M.J. (2009). Congenital and opportunistic infections: Ureaplasma species and Mycoplasma hominis. Seminars in Fetal \& Neonatal Medicine. 14, 190 199.

18. Williams, C.M.; Okada, D.M.; Marshall, J.R.; Chow, A.W. (1987). Clinical and microbiologic risk evaluation for post-cesarian section endometritis by multivariate discriminant analysis: role of intraoperative Mycoplasma, Aerobes and Anaerobes. Am. J. Obstet. Gynecol. 156, $967-$ 974.

19. Xie, X.; Zhang, J. (2006). Trends in the rates of resistance of Ureaplasma urealyticum to antibiotics and identification of the mutation site in the quinolone resistance-determining region in Chinese patients. FEMS Microbiol Lett. 259, 181-186. 